\title{
Simultaneous transmission of audio and video signals using visible light communications
}

\author{
Do Ky Son ${ }^{1,5}$, EunByeol Cho ${ }^{1}$, Inkyu Moon², Zabih Ghassemlooy ${ }^{3}$, Soeun $\mathrm{Kim}^{4}$ and Chung Ghiu Lee ${ }^{1 *}$
}

\begin{abstract}
A visible light communication system for parallel transmission of high-quality audio and video signals using illumination white and red light-emitting diodes (LEDs) is presented in this paper. For video transmission, we adopts the pulse width modulation (PWM) scheme at the carrier frequency of $15 \mathrm{MHz}$, whereas for the analog audio signal, the Sony/Philips Digital standard is used. We show that successful simultaneous high-quality audio and video signal transmissions over a link span of $50 \mathrm{~cm}$ are possible without the need for optimized optics. For audio transmission, only the link span is doubled to $100 \mathrm{~cm}$.
\end{abstract}

Keywords: Optical communication; Visible light communication (VLC); Audio transmission; Video transmission; Digital audio; PWM video; Light-emitting diodes (LEDs)

\section{Introduction}

During the last four decades, the technical progress in the development of light-emitting diodes (LEDs) has been breathtaking. State-of-the-art LEDs emitting in the visible spectrum are small, rugged, reliable, bright, and highly power efficient. Nowadays, illumination LEDs are predicted to be used in myriads of applications [1]. Since LED illumination inherits the advantages of standard LEDs, it offers numerous advantages, such as a considerable power reduction, extreme durability, size reduction, a greater degree of freedom for illumination shape, and a high resistance to shock and vibration. In addition, LEDbased lighting requires less maintenance costs due to its long lifetime, and the chip per unit price of luminous flux is continuously decreasing. Due to these characteristics, LEDs have become very attractive, not only for illumination but also for outdoor and indoor decoration and advertisement.

Besides illumination, LEDs have been finding applications in data communications due to their fast switching time. As an optical source for short-range indoor communications, visible LEDs are cheap and reliable, offering high data rate capabilities. An optical wireless communication link using high-brightness illumination LEDs has

\footnotetext{
* Correspondence: clee@chosun.ac.kr

'Department of Electronic Engineering, Chosun University, Gwangju 501-759, South Korea

Full list of author information is available at the end of the article
}

been demonstrated to support a modulation bandwidth of up to $20 \mathrm{Mb} / \mathrm{s}$ [2]. The theoretical background for visible light communication (VLC) systems is similar to that found in infrared (IR) communications except that VLCs use a much wider visible spectrum, as outlined in [3-5]. In addition, we have seen several practicalbased high-speed VLCs reported in [6-9]. Through the use of flashlight LEDs, a simplex link and a duplex link combining the two simplex links have also been reported [7].

A white LED-based VLC link was demonstrated for a $100-\mathrm{Mb} / \mathrm{s}$ non-return to zero (NRZ) data format using a low-cost equalizer [8]. A VLC link operating at $513 \mathrm{Mb} / \mathrm{s}$ based on the combination of discrete multi-tone (DMT), quadrature amplitude modulation (QAM), bit loading, and power loading, as well as with symmetrical clipping, has been reported in [9]. Application of VLCs for local area networks offering full Ethernet $10-\mathrm{Mb} / \mathrm{s}$ bit rates over a short distance has been reported in [10]. Recently, the application of LEDs to real services, such as audio and video signal transmissions has been investigated, where frequency modulation (FM) with a carrier frequency of $100 \mathrm{kHz}$ using 64 LEDs has been used for audio signals [11] and a bit rate of $2 \mathrm{Mb} / \mathrm{s}$ is employed for video broadcasting [12]. Amplitude modulation (AM) and FM have been adopted for audio and video (c) 2013 Son et al.; licensee Springer. This is an open access article distributed under the terms of the Creative Commons Attribution License (http://creativecommons.org/licenses/by/2.0), which permits unrestricted use, distribution, and reproduction in any medium, provided the original work is properly cited. 
transmissions in cable television (CATV) systems $[13,14]$, and pulse width modulation (PWM)-based video and audio signal transmissions have been reported in [15]. However, analog-based modulation schemes mostly adopted in CATV systems are not ideal for LEDbased communications. This is because the luminous intensity of LEDs does not have a linear characteristic over the entire injected forward current, becoming non-linear at higher current levels [16]. This problem is more acquitted at a higher modulation index or multiplexed analog-based system as in subcarrier intensity modulation. In addition, amplitude modulationbased systems are more vulnerable to noise than digital schemes.

Nowadays, the digital audio format based on the Audio Engineering Society-European Broadcasting Union (AES-EBU) interface is widely used in most media devices because of certain advantages [17], such as the use of a single signal to carry both data and clock information and no DC component as part of the signal power spectral density. Additionally, using the 24-bit word length makes it appropriate for professional applications $[17,18]$. A serial digital transmission of multi-channel audio signals has been reported in [19], where an analog multi-channel audio signal was converted into a digital audio signal by using a $44.1-\mathrm{kHz}$ pulse code modulation (PCM) technique.

Recently, we have seen experimental results on signal transmission demonstration based on VLC links [20-22]. A 2-Mbps VLC downlink for video broadcasting or home automation has been reported in [20], which employs a 125 -kbps infrared uplink channel for commands. A realtime eight-channel audio data has been experimentally demonstrated based on an optical code-division multiple access (OCDMA) system using VLC link [21]. Also, a three-terminal high-speed optical wireless demonstrator was reported in [22] that supports video streams with a data rate of up to $4 \mathrm{Mb} / \mathrm{s}$.

In this paper, we present the development of VLC for audio and video transmissions using white and red LEDs for home audio-video systems delivering highquality signals. We have used the Sony/Philips Digital Interconnect Format (S/PDIF) standard format [23], which is a modification from the AES/EBU format, and the naturally sampled PWM format for audio and video signals, respectively.

The paper is organized as follows. Section 2 describes the audio and video signal formats. The development of the transmitter and receiver modules is described in Section 3, including the specifications of the main components. Section 4 presents the experiment and measurement results. The technical aspects related to the applications are discussed in Section 5. The paper is concluded in Section 6.

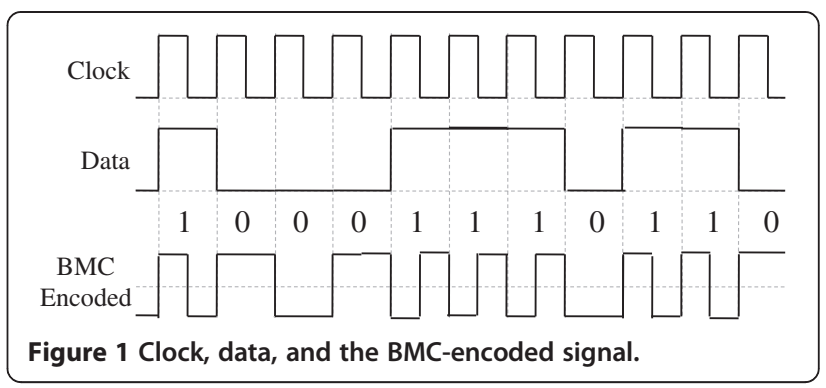

\section{Signal modulation format 2.1 Audio signal}

We have used S/PDIF (more commonly known as Sony Philips Digital Interface) [23], a widely adopted standard format used in digital audio transmission, especially in home media entertainment devices. S/PDIF is a data link layer protocol, which contains a set of physical layer specifications for carrying digital audio signals between devices and components over either optical or electrical cables. S/PDIF is standardized in IEC 60958 as IEC 60958 type II (IEC 958 before 1998) and is essentially a minor modification of the original AES/EBU standard (used for digital audio signal transport) [17] for consumer electronics, employing small differences in the protocol and requiring less expensive hardware. In S/ PDIF, the digital data stream is encoded using the bi-phase mark code (BMC) also known as the differential Manchester encoding, which is a kind of phase modulation in which clock and data signals are combined to form a single two-level self-synchronizing bit stream (see Figure 1), where the level change occurs at the beginning of every bit period. It is a differential encoding scheme, using the presence or absence of transitions to indicate a logical value. Logics 1 and 0 are represented by mid-bit level and no mid-bit level changes, respectively, thus offering built-in synchronization capabilities.

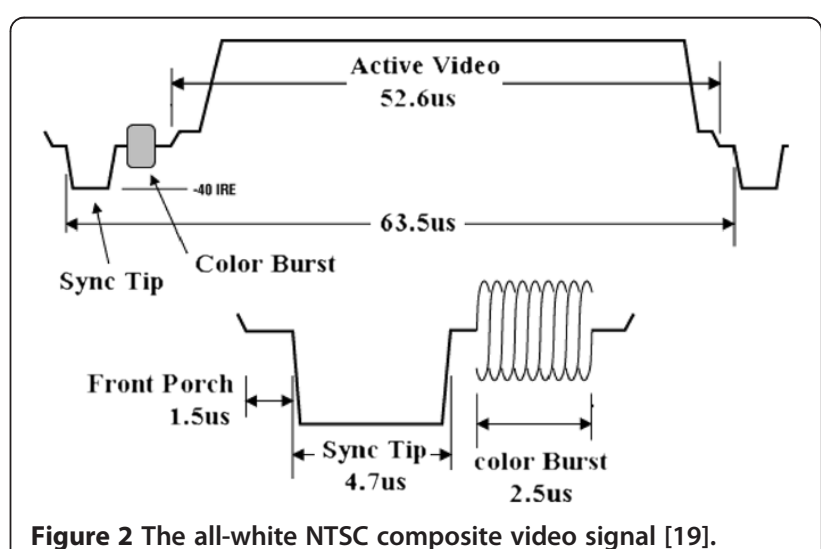



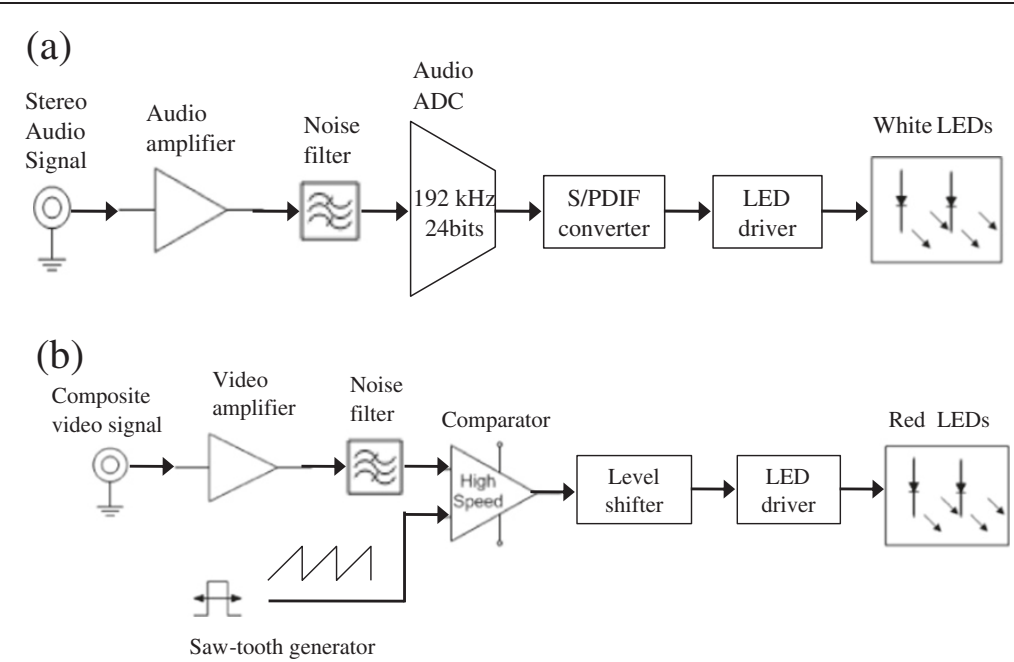

Figure 3 The transmitter system block diagrams: (a) audio and (b) video.

\subsection{Video signal}

Analog TVs adopt a composite video signal, the commonly used analog video interface [24]. Composite video is also referred to as CVBS (color, video, blanking, and sync) or the composite video baseband signal, which combines the brightness information (luma), the color information (chroma), and the synchronizing signals on just one line (see Figure 2) [24].

There are three standard analog TV formats such as Phase Alternating Line (PAL), National Television System Committee (NTSC), and Sequential Color with Memory (SECAM). PAL uses 4.43-MHz, NTSC uses 3.58-MHz, and SECAM uses $4.53-\mathrm{MHz}$ [25] color subcarriers. A very high bit rate is required if we use analog-to-digital conversion $(\mathrm{ADC})$ for the video transmission. For example, with an 8-bit ADC, the sampling rate must be $10 \mathrm{MS} / \mathrm{s}$ and the resulting output data rate from the ADC for video

Table 1 Summary of system parameters

\begin{tabular}{ll}
\hline Parameters & Values \\
\hline Semi-angle at half power of LEDs & $50^{\circ}$ \\
Red LED power & $50 \mathrm{~mW}$ \\
White LED power & $100 \mathrm{~mW}$ \\
Audio signal transmitter & $4 \times 5$ white LED using S/PDIF \\
Video signal transmitter & $5 \times 5$ red LED using PWM \\
ADC & $192-\mathrm{kHz}$ sampling rate, 24 bits \\
PWM frequency (period) & $15 \mathrm{MHz}$ (66.7 ns) \\
Audio receiver & $15-\mathrm{Mb} / \mathrm{s}$ PD with a sensitivity \\
Video receiver & of $-24 \mathrm{dBm}$ \\
& $50-\mathrm{Mb} / \mathrm{s}$ PD with a sensitivity \\
Transmitter module spacing & of $-17.5 \mathrm{dBm}$ \\
Transmission span & $10 \mathrm{~cm}$ \\
\hline
\end{tabular}

transmission will be $80 \mathrm{Mb} / \mathrm{s}$. The maximum modulation frequency of the ordinary high-brightness LED is around $20 \mathrm{MHz}$, Which is not sufficient to support $80 \mathrm{Mb} / \mathrm{s}$. Therefore, one solution would be to employ pulse time modulation schemes such as PWM, pulse frequency modulation (PFM), square wave FM, etc. [26].

\section{System description}

\subsection{Transmitter}

The schematic diagrams of audio and video transmitter modules are illustrated in Figure 3. In Figure 3a, the analog audio signal is amplified and digitized prior to being converted into an S/PDIF format. The S/PDIF audio signal drives a $4 \times 5$ white LED cluster made up of blue LEDs with yellow phosphor. In Figure $3 \mathrm{~b}$, the amplified analog video signal that passed through a bandpass filter is compared with the reference sawtooth carrier

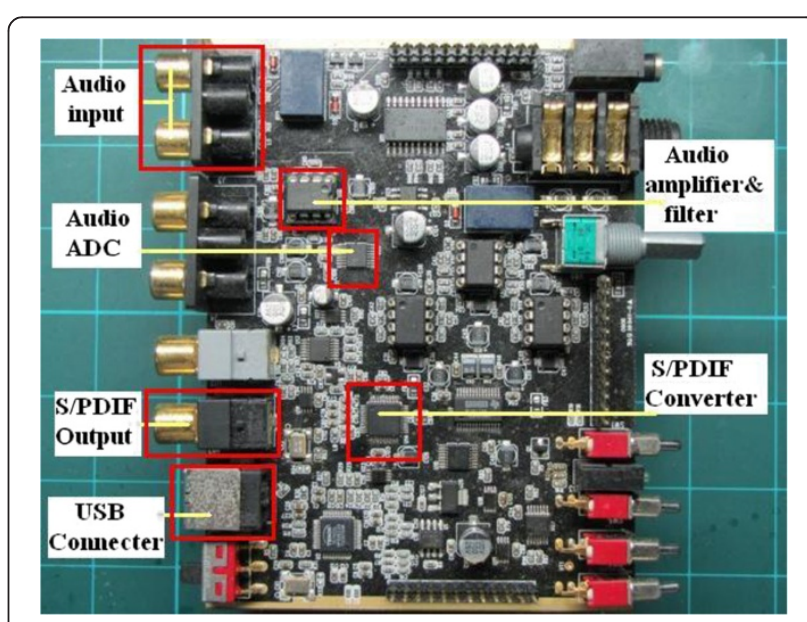

Figure 4 The audio transmitter module. 


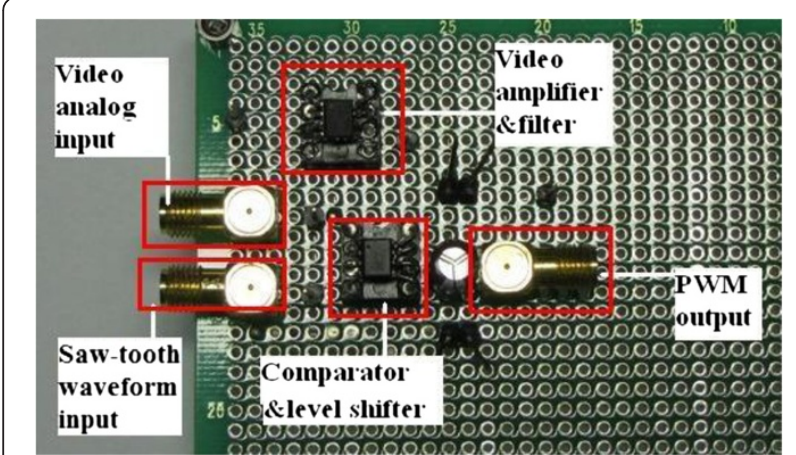

Figure 5 The video transmitter module. signal, which generates the naturally sampled PWM signal that drives a $5 \times 5$ red LED cluster. The colors of the LEDs for each signal depend on the transmitted optical power. The semi-angle at half power of the LEDs, $\theta_{1 / 2}$, is $50^{\circ}$ (Table 1 ).

The output of the ADC is an Inter-IC Sound (I2S) signal, which includes a master clock, bit clock, left right clock, and data clock [19]. The I2S signal is then converted to the S/PDIF signal. The S/PDIF signal data stream consists of a series of 1 and 0 digital bits that describe the audio waveforms [23]. The color subcarrier frequencies, $f_{\mathrm{c} \text {-sub }}$, of the analog video signal are 4.43361875 and $3.579545 \mathrm{MHz}$ for PAL and NTSC,

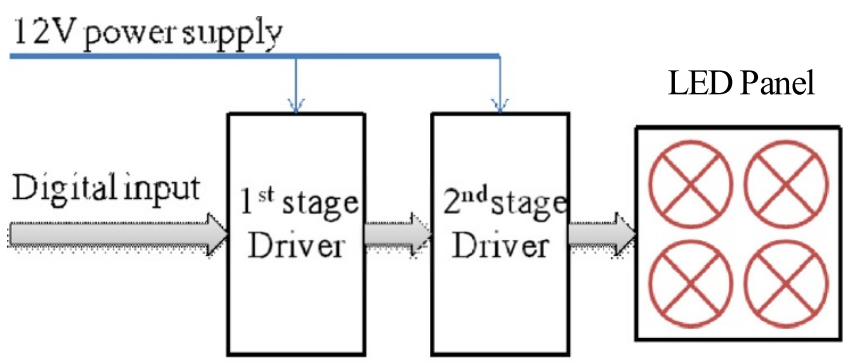

(a)

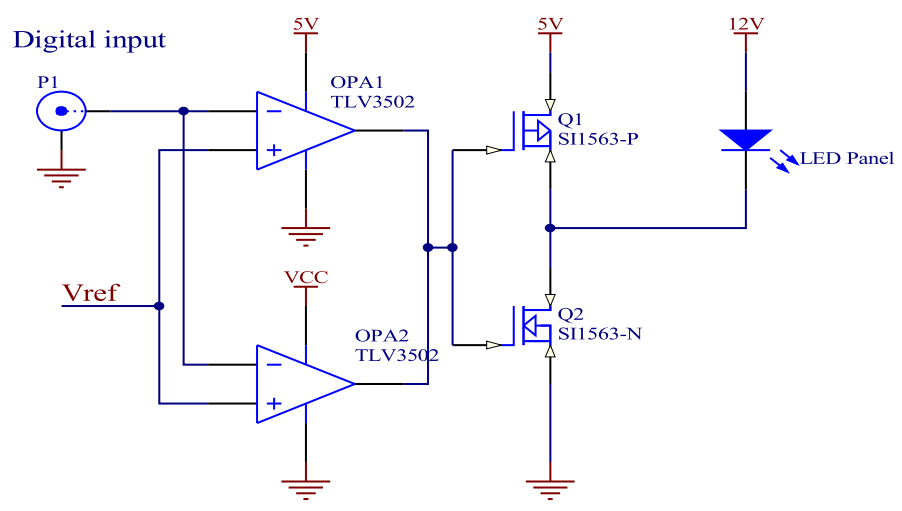

(b)

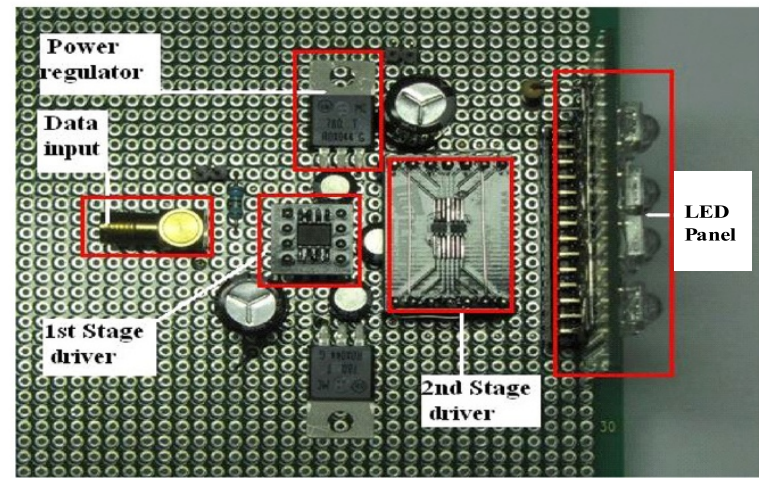

(c)

Figure 6 LED driver: (a) block diagram, (b) circuit diagram, and (c) board. 


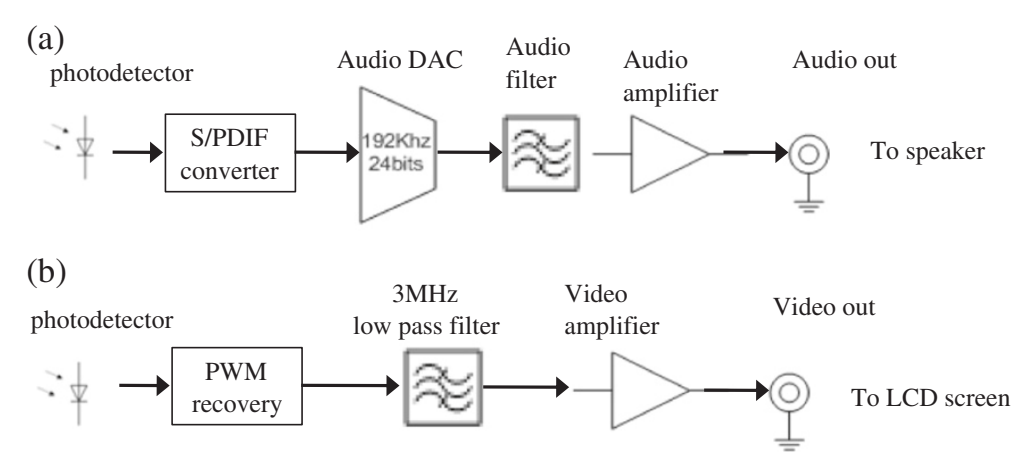

Figure 7 The block diagrams of the receiver modules: (a) audio and (b) video.

respectively [25]. The composite video signal following amplification and filter is compared with the linear ramp carrier signal at a frequency of $15 \mathrm{MHz}$, which is only four and five times the $f_{\mathrm{c} \text {-sub }}$, using a high-speed comparator (with the propagation delay and rise/fall times of 4.5 and $1.5 \mathrm{~ns}$, respectively).
Figures 4 and 5 show the audio and video transmitter modules.

The transmission distance between the LEDs and the optical receiver was measured at the point where the video and audio play worked well. Of course, the link span is dependent on the optical transmit power and

(a)

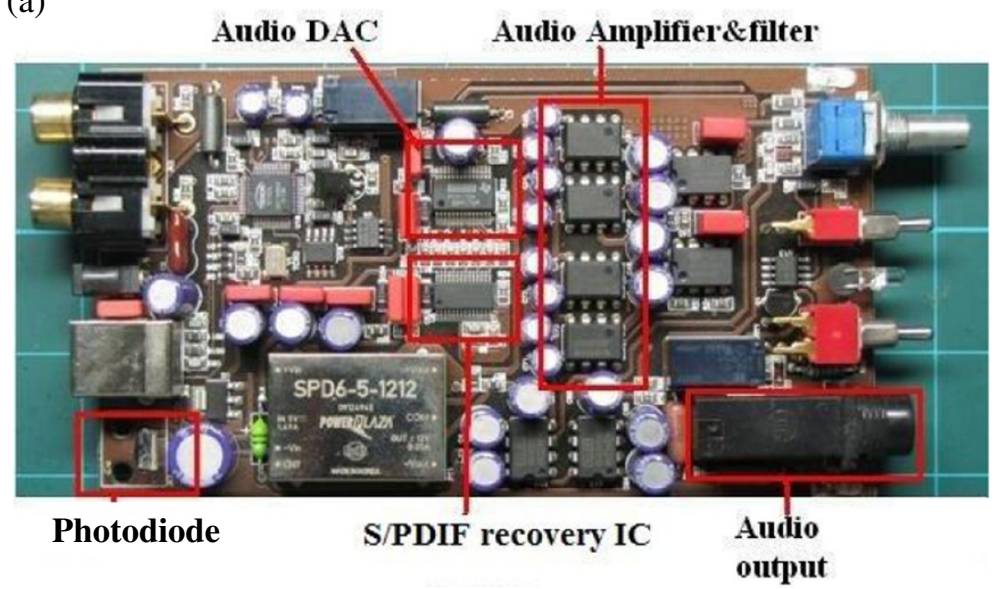

(b)

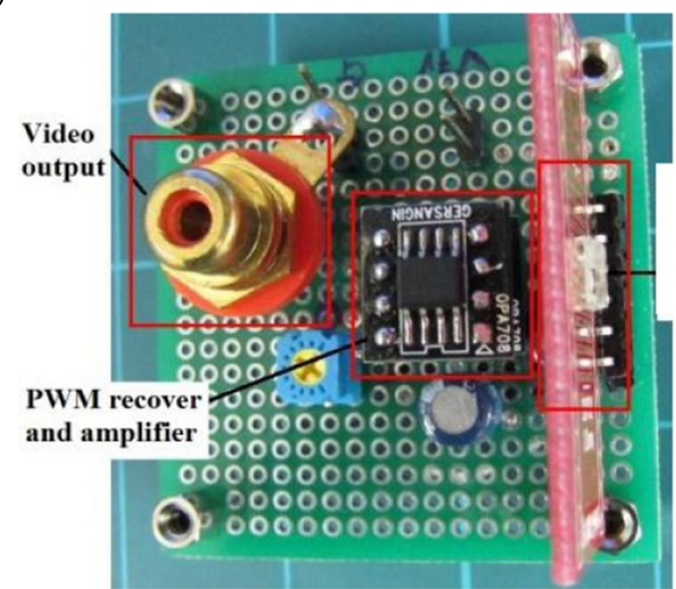

Photodiode

Figure 8 The receiver modules: (a) audio and (b) video. 


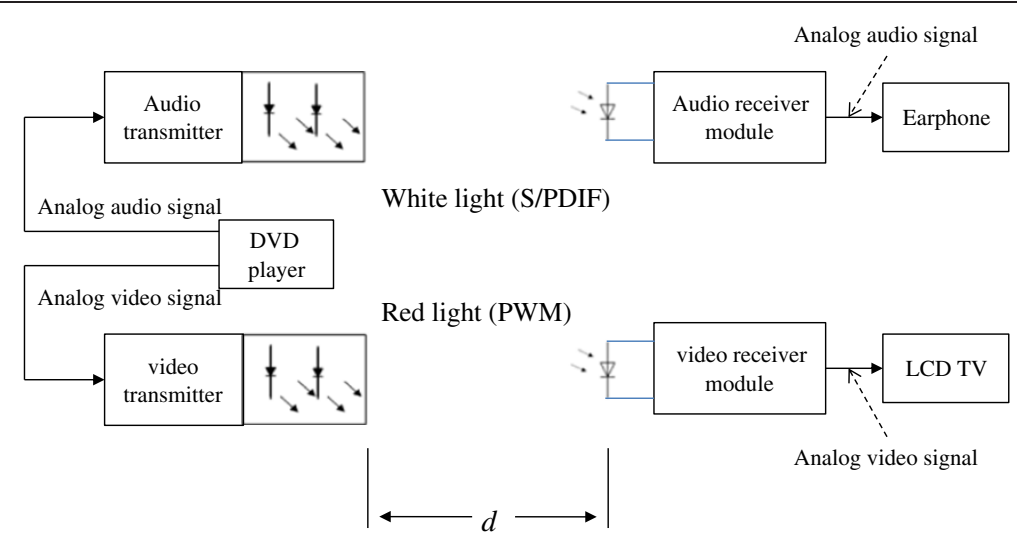

Figure 9 The experimental setup.

the photodetector (PD) receiver sensitivity. We have designed an LED driver module, which supports a maximum power of $500 \mathrm{~mW}$. The schematic diagram of the LED driver is shown in Figure 6a. At the first stage, two high speed comparators (TLV3502) with a maximum output current of $74 \mathrm{~mA}$ function as a NOT gate with two inverted output signals. The output of the first stage drives the second stage driver where a fast switching power MOSFET (Si1563 from Vishay, Malvern, PA, USA) is used to drive the LED panel. A push-pull configuration is adopted for the second stage in order to supply a maximum of $500 \mathrm{~mW}$ to the LED panel. The circuit diagram and the driver board of the LED driver are shown in Figure 6b,c, respectively.

\subsection{Receiver modules}

Figure 7 shows the block diagrams of the receiver for both audio and video signals. Two different types of PDs were used for the audio and video signals. For the audio part, the output of a low-speed PD (i.e., $15 \mathrm{Mb} / \mathrm{s}$ with a receiver sensitivity of $-24 \mathrm{dBm}$ ) is first passed through the S/PDIF module followed by a high-quality stereo audio digital-to-analog converter (DAC) module. We have chosen a 192-kHz, 24-bit advanced segment stereo DAC for a high-quality audio signal. Filtering and amplification ensures the required audio output level. For the video link, a high-speed PD (i.e., $50 \mathrm{Mb} / \mathrm{s}$ with a receiver sensitivity of $-17.5 \mathrm{dBm}$ ) followed by the PWM demodulator, a $3-\mathrm{MHz}$ filter, and a video amplifier are used to recover the video signal. Figure 8 shows the audio and video receiver modules.

There are a number of ICs available for the audio ADC. Here we have chosen a very simple chip (AK5386 from Asahi-Kasei, Chiyoda, Tokyo, Japan), which is a stereo A/D converter with a wide-ranging sampling rate from 8 to $216 \mathrm{kHz}$, widely used in both consumer and

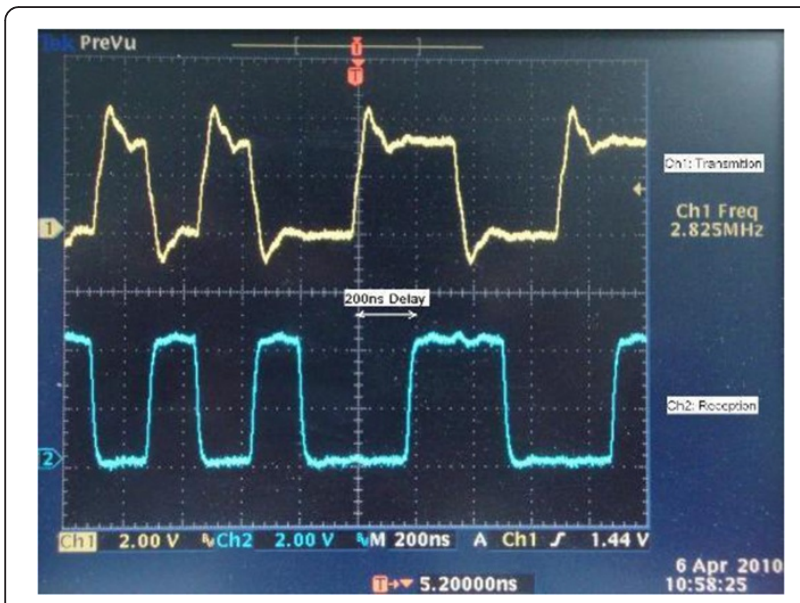

Figure 10 The digital audio signal waveforms: input (upper trace) and output (lower trace) of the S/PDIF module.

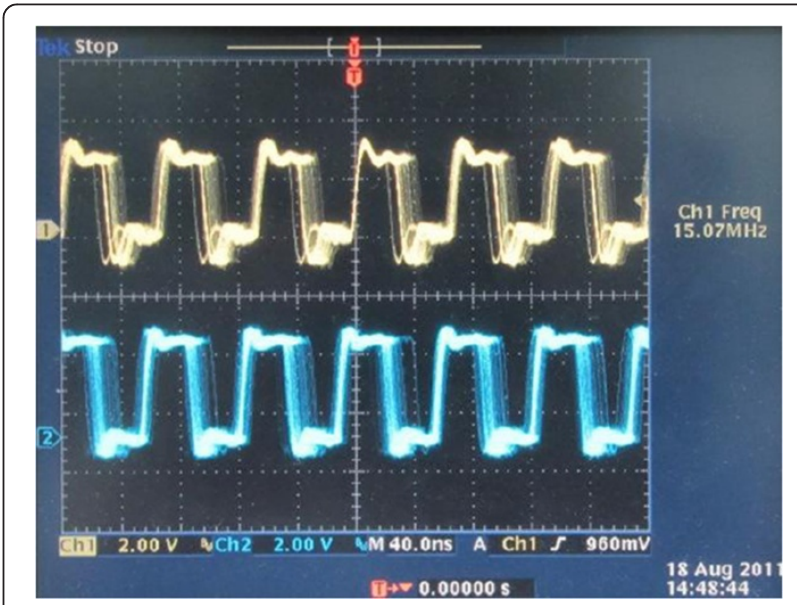

Figure 11 The PWM video signal waveforms at the input (upper trace) and output (lower trace) stages. 


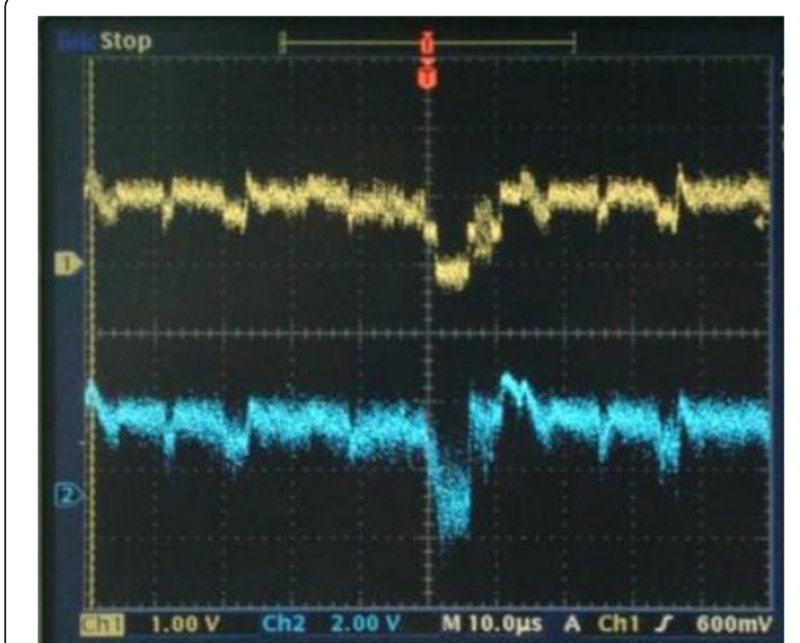

Figure 12 The analog video signal waveforms: input (upper trace) and output (lower trace).

professional audio systems. The technology is based on enhanced dual-bit $\Delta \Sigma$ scheme, thus offering a high accuracy and a low cost.

\section{Experiments and results}

The transmission of audio and video signals using the developed modules has been demonstrated experimentally. The experimental setup is shown in Figure 9. A DVD player is used to generate both the audio and video signals. The white and red light sources are used for transmission of audio and video signals, respectively. At the receiver, LCD TV and speakers are used to monitor the quality of the video and audio signals following postphotodetection processing. As shown in Figure 9, transmitters and receivers were placed in a directed diffuse link configuration [3], with $10-\mathrm{cm}$ spacing between the two transmitter modules. The transmission span was set at $50 \mathrm{~cm}$ with no lenses. The link distance can be extended by using appropriate optics both at the source and the receiver or increasing the transmit power within the eye safety limit.

Figure 10 shows the digital audio signal S/PDIF waveforms at the input (upper trace) and output of the S/PDIF module (lower trace) stages. Due to the response time of the used devices, the total transmission delay achieved is $200 \mathrm{~ns}$, which is sufficiently small and therefore does not affect the audio and video signals quality. Figure 11 illustrates the PWM video signal waveforms at the input (upper trace) and output (lower trace) stages.

In order to assess the transmission quality of the video signal, we used two test signals: (1) the NTSC composite video time domain waveform (see Figure 12) with the upper and lower traces showing the input and recovered analog video signals and (2) the video test cards (i.e., SMPTE color bars [16] and a modified version of the PM5544 Philips test card) as shown in Figure 13. As shown in Figure 13, the slight difference in the contrast between received and transmitted video images might be due to contribution (leak) from the audio signal. However, this can be improved by using an optical band-pass filter and higher carrier frequency for PWM. (a)

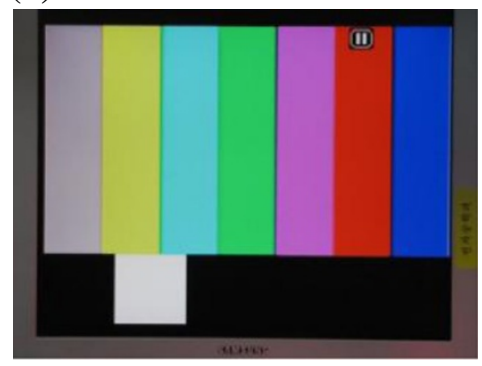

(c)

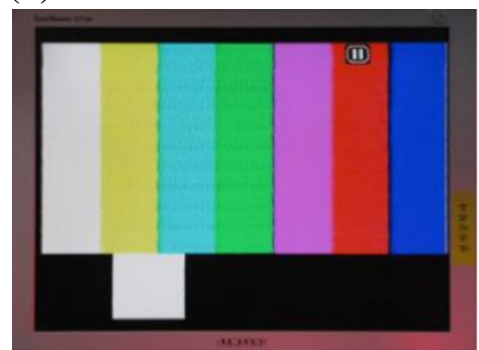

(b)

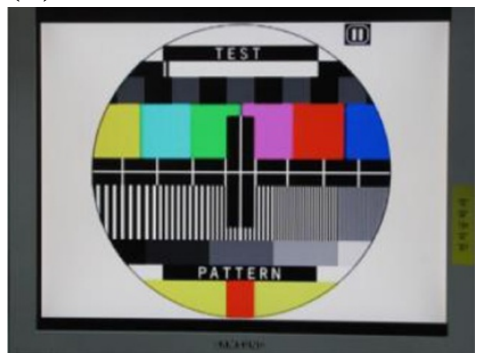

(d)

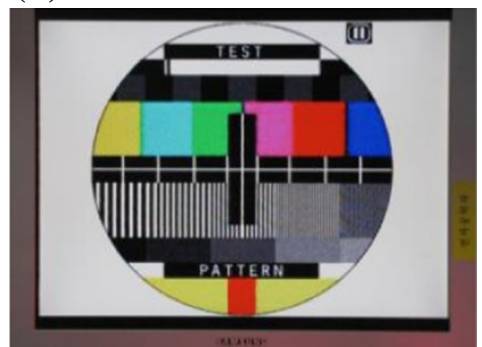

Figure 13 The video signal test cards. $(\mathbf{a}, \mathbf{b})$ The original images before transmission. $(\mathbf{c}, \mathbf{d})$ The recovered images at the output of the receiver. 


\section{Discussions}

In order to improve the video quality, an ADC can be used for the digital conversion of the video signal and then the digital video signal can be transmitted via LEDs. By adopting some compression algorithms, one can reduce the data rate of the digital video signal and avoid using expensive high-speed ADCs. We practically demonstrated parallel transmission of audio and video signals using LEDs. For the audio link, the S/PDIF format was adopted to allow transmission of $5.1,7.1$, or 9.1 digital audio signals, which is very attractive for home entertainment applications.

Since the PDs used have their peak in the red wavelength region, then the maximum transmission span achieved was based on the red LED output. The links were fully tested for the quality of transmission using the standard test cards for video and audio signals. Additional information such as time information or the name of the song or video in multi-channel applications could be added to both audio and video signals. The proposed system is attractive for home entertainment application, museums, galleries, etc.

\section{Conclusions}

A visible light communication system for transmission of audio and video signals employing visible LEDs was presented. The S/PDIF formatted audio signal is digitized and used to intensity-modulate white illumination LEDs. Simultaneously, the analog video signal was transmitted using PWM via red illumination LEDs. The transmission and signal recovery were successful at the transmission distance of $50 \mathrm{~cm}$ without optical filters. The system quality was assessed using standard test signals for both links as well as by viewing the video signal and listening to the audio signal. It is expected that the proposed system could be adopted for a number of applications where high-quality sound and vision are required.

\section{Competing interests}

The authors declare that they have no competing interests.

\section{Acknowledgements}

This study was supported in part by research funds from Chosun University, 2012.

\footnotetext{
Author details

'Department of Electronic Engineering, Chosun University, Gwangju 501-759, South Korea. ${ }^{2}$ School of Computer Engineering, Chosun University, Gwangju 501-759, South Korea. ${ }^{3}$ Optical Communications Research Group, Faculty of Engineering and Environment, Northumbria University, Newcastle NE1 8ST, UK. ${ }^{4}$ Advanced Photonics Research Institute, Gwangju Institute of Science and Technology, Gwangju 500-712, South Korea. ${ }^{5}$ Max Speed Media and Technology, Hanoi, Vietnam.
}

Received: 19 April 2013 Accepted: 13 October 2013

Published: 28 October 2013

\section{References}

1. EF Schubert, Light-Emitting Diodes, 2nd edn. (Cambridge University Press, Cambridge, 2006)

2. CG Lee, CS Park, J-H Kim, D-H Kim, Experimental verification of optical wireless communication link using high-brightness illumination light-emitting diodes. Opt. Eng. 46(12), 125005 (2007)

3. JM Kahn, JR Barry, Wireless infrared communications. Proc. IEEE 85(2), 265-298 (1997)

4. Z Ghassemlooy, WO Popoola, S Rajbhandari, Optical Wireless Communications: System and Channel Modelling with MATLAB ${ }^{\circledR}$ (CRC Press, Boca Raton, 2012)

5. T Komine, M Nakagawa, Fundamental analysis for visible-light communication system using LED lights. IEEE T. Consum. Electr. 50(1), 100-107 (2004)

6. J Grubor, S Randel, K-D Langer, JW Walewski, Broadband information broadcasting using LED-based interior lighting. J. Lightwave Technol. 26, 3883-3892 (2008)

7. TDC Little, P Dib, K Shah, N Barraford, B Gallagher, Using LED lighting for ubiquitous indoor wireless networking, in IEEE International Conference on Wireless and Mobile Computing, Networking and Communications 2008, WIMOB '08 (IEEE, Piscataway, 2008), pp. 373-378

8. HL Minh, D O'Brien, G Faulkner, L Zeng, K Lee, D Jung, Y Oh, ET Won, 100 $\mathrm{Mb} / \mathrm{s}$ NRZ visible light communications using a postequalized white LED. IEEE Photonic Tech. L. 21(15), 1063-1065 (2009)

9. J Vucic, C Kottke, S Nerreter, K-D Langer, JW Walewski, 513 Mbit/s visible light communications link based on DMT-modulation of a white LED. J. Lightwave Technol. 38(24), 3512-3518 (2010)

10. PA Haigh, TT Son, E Bentley, Z Ghassemlooy, H Le Minh, L Chao, Development of a visible light communications system for optical wireless local area networks, in IEEE Computing, Communications and Applications Conference (Com ComAp) (IEEE, Piscataway, 2012), pp. 351-355

11. G Pang, C-H Chan, K-L Ho, T Kwan, E Yang, Visible light communication for audio systems. IEEE T. Consum. Electr. 45(4), 1112-1118 (1999)

12. J Rufo, J Rabadan, F Delgado, C Quintana, R Perez-Jimenez, Experimental evaluation of video transmission through LED illumination devices. IEEE Trans. Consum. Electr. 56(3), 1411-1416 (2010)

13. JA Chiddix, HE Laor, DM Pangrac, LD Williamson, RW Wolfe, AM video on fiber in CATV systems. IEEE J. Sel. Area Comm. 8(7), 1229-1239 (1990)

14. K Kikushima, K Suto, H Nakamoto, H Yoshinaga, C Kishimoto, M Kawabe, K Kumozaki, N Shibata, Super-wide-band optical FM modulation scheme and its application to multichannel AM video transmission systems. IEEE Photonic Technol 8(6), 839-841 (1996)

15. Z Ghassemlooy, B Wilson, Optical PWM data link for high quality video and audio signals. IEEE T. Consum. Electr. 40(1), 55-63 (1994)

16. S King, Luminous intensity of an LED as a function of input power. ISB J. Phys 2(2), Paper number 3 (2008)

17. M Angelici, M Bianchessi, SD Feste, N Serina, New architecture for an AES-EBU digital audio receiver. IEEE T. Consum. Electr. 43(3), 694-698 (1997)

18. F Rumsey, J Watkinson, Digital Interface Handbook, 3rd edn. (Focal Press, Burlington, 2003)

19. M Song, $O$ Kwon, $Y$ Chung, A serial connection technique of speakers for multi-channel audio systems. IEEE T. Consum. Electr. 51(2), 611-616 (2005)

20. F Delgado, I Quintana, J Rufo, JA Rabadan, C Quintana, R Perez-Jimenez, Design and implementation of an Ethernet-VLC interface for broadcast transmissions. IEEE Commun. Lett. 14(12), 1089-1091 (2010)

21. MF Guerra-Medina, O Gonzalez, B Rojas-Guillama, JA Martin-Gonzalez, F Delgado, J Rabadan, Ethernet-OCDMA system for multi-user visible light communications. Electron Lett. 48, 227-228 (2012)

22. D O'Brien, R Turnbull, HL Minh, G Faulkner, O Bouchet, P Porcon, ME Tabach, E Gueutier, M Wolf, L Grobe, J Li, High-speed optical wireless demonstrators: conclusions and future directions. J. Lightwave Technol. 30, 2181-2187 (2012)

23. Wikipedia, S/PDIF, 2013. http://en.wikipedia.org/wiki/S/PDIF. Accessed 23 Oct 2013

24. MAXIM, Video Basics, Application Note 734, 2001. http://www. maximintegrated.com/app-notes/index.mvp/id/734. Accessed 23 Oct 2013

25. National Television System Committee, 2012. http://www.ntsc-tv.com/. Accessed 23 Oct 2013

26. B Wilson, Z Ghassemlooy, Pulse time modulation techniques for optical fibre communications, in Analogue Optical Fibre Communication, ed. by $B$ Wilson, Z Ghassemlooy, I Darwazeh (IEE, London, 1995), pp. 89-117

doi:10.1186/1687-1499-2013-250

Cite this article as: Son et al:: Simultaneous transmission of audio and video signals using visible light communications. EURASIP Journal on Wireless Communications and Networking 2013 2013:250. 